\title{
Damage identification of low-density material-filled sandwich panels with truss core based on vibration properties
}

\author{
Jie Le ${ }^{1,2}$, Lingling Lu ${ }^{1,3}$, Yabo Wang ${ }^{1,3}$, Hongwei Song ${ }^{1,3}$, Xiaodong Xing ${ }^{2}$ \\ and Chenguang Huang ${ }^{1,3}$
}

\begin{abstract}
A damage-identification method based on flexibility matrix and Teager energy operator is proposed for low-density material-filled sandwich panels with truss core. In the proposed damage index, weight coefficient $r$ is introduced to consider the effect of damages on both high-order and low-order modes. Numerical simulations and experiments are conducted to assess the performance of the proposed method. Effects of Young's modulus of the filler material on the accuracy of the proposed method are also discussed. Results reveal that the method is reliable and effective for singledamage and multiple-damages identification of filled sandwich panels with truss core, and weight coefficient plays an important role, especially for cases with multiple damages or damages of small extent. Damage identification becomes more difficult as Young's modulus of the filler increases, and there is a critical value, after which the damage could not be identified by the proposed method.
\end{abstract}

\section{Keywords}

Filled sandwich panels with truss core, damage identification, Teager energy operator, flexibility matrix, filler's influence

\section{Introduction}

As a class of newly developed lightweight multifunctional structure, sandwich panel with truss core (SPTC) is made of two thin face-sheets and one thick lightweight core with various truss configuration, for example, diamond, tetrahedron, kagome, and hourglass. ${ }^{1-3}$ This special structural configuration endows the SPTC with many superior properties such as high specific bending stiffness, sound-shock resistance, good thermal insulation, and acoustical isolation. ${ }^{4-7}$ For example, it has been considered as one of the most promising candidates to be applied in the thermal protection system (TPS) of high-speed aircrafts. However, various damages or defects may occur in the SPTC during manufacturing process or being in service, including breakage of truss member, burn-through of face-sheets, buckling of the panel, and so on. ${ }^{8,9}$ These damages and defects may change the mechanical properties of the SPTC ${ }^{10}$ and increase the risk of structure failure. Therefore, damage detection in the early stage is of great importance in avoiding catastrophic events. In practical applications, low-density or porous functional material are often filled in the void space of the SPTC between the two face-sheets, in order to further enhance the performance in shock resistance, ${ }^{1,12}$ thermal insulation, or acoustical isolation. ${ }^{13,14}$ The filled material, which may additionally hinder direct visual inspection and significantly alter the dynamic behavior, makes damage identification of the SPTC even difficult.

Recently, non-destructive testing (NDT) methods are developed to detect damages in various sandwich structures, such as acoustic emission (AE), ${ }^{15,16}$ electrical impedance, ${ }^{17}$ thermography, ${ }^{18-20}$ and guided wave. ${ }^{21-23}$

\footnotetext{
'Key Laboratory for Mechanics in Fluid Solid Coupling Systems, Institute of Mechanics, Chinese Academy of Sciences, Beijing, China

${ }^{2}$ School of Mechanical and Electrical Engineering, Harbin Engineering University, Harbin, China

${ }^{3}$ School of Engineering Science, University of Chinese Academy of Sciences, Beijing, China
}

\section{Corresponding author:}

Lingling Lu, Key Laboratory for Mechanics in Fluid Solid Coupling Systems, Institute of Mechanics, Chinese Academy of Sciences, No. 15 Beisihuanxi Road, Beijing 100190, China.

Email: lulingling@imech.ac.cn 
For sandwich composite structures, Sikdar et al. ${ }^{16}$ propose an AE-based real-time health-monitoring framework to identify the probable damage locations. Wong et al. ${ }^{17}$ used an electrical impedance resonance shift method to identify the defect accurately in honeycomb core sandwich structures because damages can cause a disrupted change of imaginary part of electrical impedance. Li et al. ${ }^{21}$ proposed a novel multi-dimensional signal-processing and mode-tracking approach with a reference-free perspective for the damage identification and localization in the media with large attenuation fact. And the results reflected that the methods offered high accuracy for locating damage positions.

Besides, some vibration-based methods ${ }^{24,25}$ are recently introduced to inspect the health status of the sandwich structures. Lestari and $\mathrm{Qiao}^{26}$ used variation of dynamic responses between healthy and damaged structures to identify damages of fiber-reinforced polymer sandwich composites. Tian et al. ${ }^{27}$ utilized uniform load surface (ULS) curvature to detect delamination defects of the lattice sandwich plate. For honeycomb sandwich beams, Zhu et al. ${ }^{28}$ propose a vibration-based method by using the frequency response function (FRF) measured at one point. Seguel and Meruane ${ }^{29}$ used high-speed three-dimensional (3D) digital image correlation (DIC) system to identify the debonding of the honeycomb sandwich panel. For composite SPTCs, Li et al. ${ }^{30}$ propose a ULS-based method, gapped smoothing method (GSM), and Teager energy operator (TEO). Lu et al..$^{31}$ propose a baseline-free identification method to identify damages in metallic SPTCs, which utilized the flexibility matrix and GSM. Later on, an improved method was also presented to identify unbound nodes damages. ${ }^{32}$

Recently, low-density thermal insulation material has been filled in the SPTC, and it is found that the filled SPTC has much better performance in thermal insulation, especially in the cases of ultra-high local heat flux, for example, irradiated by high-power laser. ${ }^{33,34}$ Compared with plates and pipes, the filled SPTC is more complicated, which consists of two facesheets, truss core, and filled material. The traditional NDT methods may not work, such as AE and guided wave. Besides, there are many kinds of filled material, such as polymer or metallic foams, porous ceramic, and silicone resin and carbon powder. The material property of the filled material may influence the effectiveness of the damage-identification method. Up to now, there is few, even no, reported work on the damage identification of the low-density material-filled SPTC.

In this article, a vibration-based damage-identification method is proposed for low-density material-filled SPTCs. The damage index $D I T_{r}$ is based on the variation of flexibility matrix, and $r$ is a weight coefficient, which is to consider effects of damages on both loworder and high-order modes. Numerical simulations and experiments are conducted to investigate effectiveness of the method in identification of single damage and multiple damages with different extents, and the effect of the Young's modulus of the filler is also discussed.

\section{Damage-identification method}

The flexibility matrix $F$ of a structural system with $n$ degrees-of-freedom can be expressed as

$$
F=\sum_{p=1}^{n} \frac{\Phi_{p} \Phi_{p}^{T}}{\omega_{p}^{2}}
$$

where $\omega_{p}$ and $\Phi_{p}$ are the $p$ th natural frequency and mode shape, respectively. The contribution of $p$ th mode on the flexibility matrix $F_{p}$ can be written as

$$
F_{p}=\frac{\Phi_{p} \Phi_{p}^{T}}{\omega_{p}^{2}}
$$

Take $M D(i)$ as the modal deflection at point $i$ under uniform unit load all over the structure, for a linear system, it can be approximately deduced as

$$
M D(i)=\sum_{p=1}^{n} \frac{\Phi_{p}(i) \sum_{j=1}^{n} \Phi_{p}(j)}{\omega_{p}^{2}}
$$

For a linear system, the definition of ULS is expressed as the deflection vector of the structure under uniform load

$$
M D=\{M D(i)\}=\sum_{p=1}^{n} F_{p} I=\sum_{p=1}^{n} M D_{p}
$$

where $I$ is $\{1, \ldots, 1\} T 1 \times n . M D_{p}$ is the $p$ th-order mode contribution to $M D$.

When a specimen is damaged, the vibration characteristics would change. In the work, variation of $M D_{p}$ is defined as

$$
\begin{aligned}
D I & =\sqrt{\sum_{p=P_{1}}^{P_{2}}\left(M D_{p D}-M D_{p U}\right)^{2}} \\
& =\sqrt{\sum_{p=P_{1}}^{P_{2}}\left(\left[F_{p} \cdot I\right]_{D}-\left[F_{p} \cdot I\right]_{U}\right)^{2}}
\end{aligned}
$$

where $P_{1}$ and $P_{2}$ are the beginning- and the endingorder modes in the practical case. $M D_{p D}$ and $M D_{p U}$ 
denote the $p$ th component of modal deflection of the damaged and undamaged models, respectively.

According to the definition of $M D_{p}$, weight of loworder modes is larger than that of high-order modes. However, higher-order modes are more sensitive to local damages. ${ }^{35}$ To identify various damages, the damage index $D I_{r}$ is defined

$$
D I_{r}=\sqrt{\sum_{p=P_{1}}^{P_{2}} \alpha_{p}^{r}\left(M D_{p D}-M D_{p U}\right)^{2}}
$$

where $\alpha_{p}^{r}$ is expressed as the weight coefficient of the pth component

$$
\alpha_{p}^{r}=\frac{\omega_{p}^{r}}{\sum_{p=P_{1}}^{P_{2}} \omega_{p}^{r}}
$$

$\alpha_{p}^{r}$ could determine the weights of low-order and high-order modes in $D I_{r}$, and $r$ is an integer. When $r$ is less than 2, the weight of low-order modes in $D I_{r}$ is larger than that of high-order modes. When $r$ is equal to 2, the weight of low-order modes in $D I_{r}$ is equal to the weight of high-order modes. When $r$ is larger than

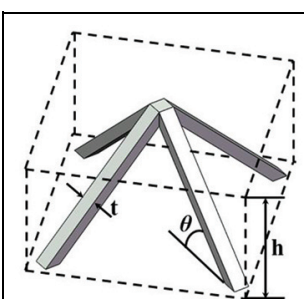

(a)

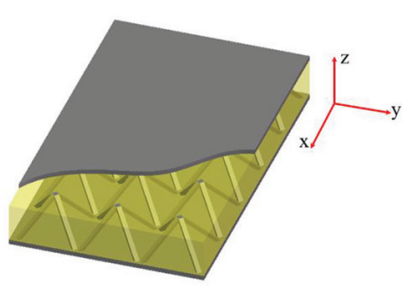

(b)
Figure I. Model information: (a) unit cell of pyramidal truss and (b) the filled sandwich panel.

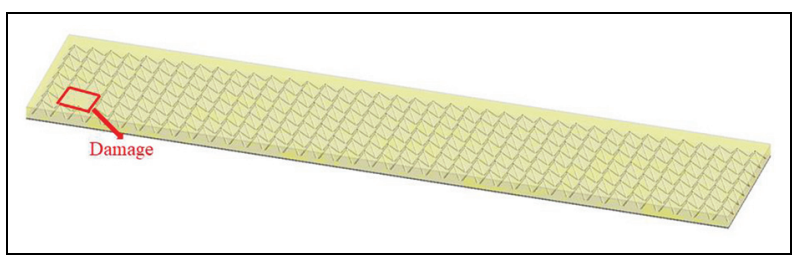

Figure 2. Example of damaged specimen.

2, the weight of low-order modes in $D I_{r}$ is smaller than that of high-order modes.

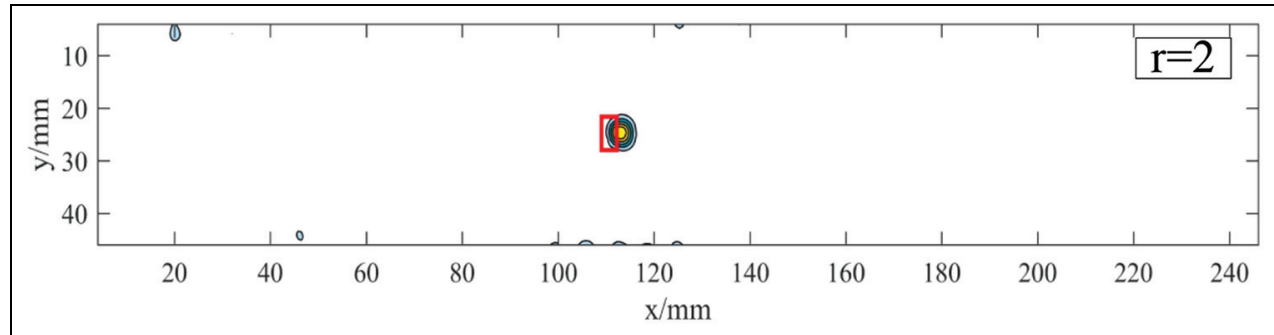

(a)

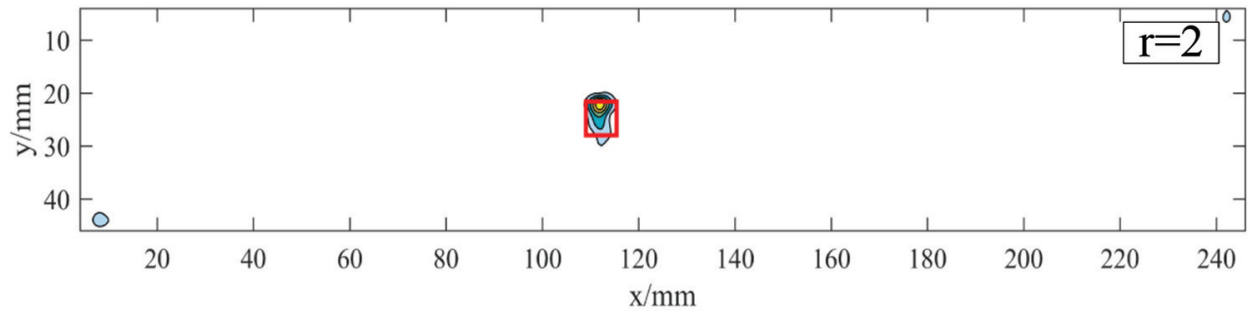

(b)

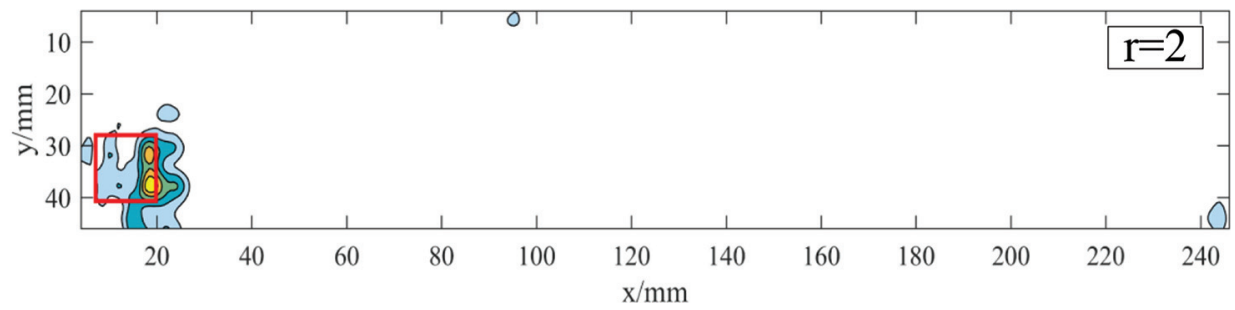

(c)

Figure 3. Results of $D I T_{2}$ : (a) $\mathrm{SI}$, (b) $\mathrm{S} 2$, and (c) $\mathrm{S} 3$. 


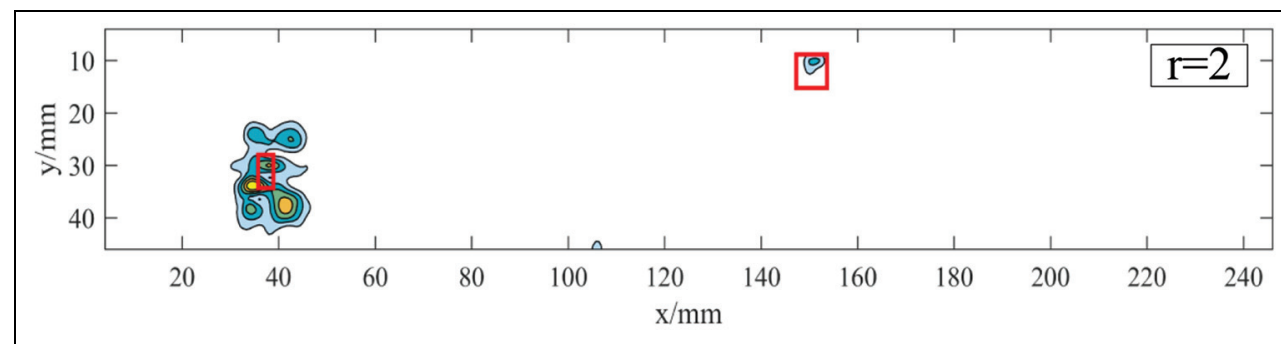

(a)

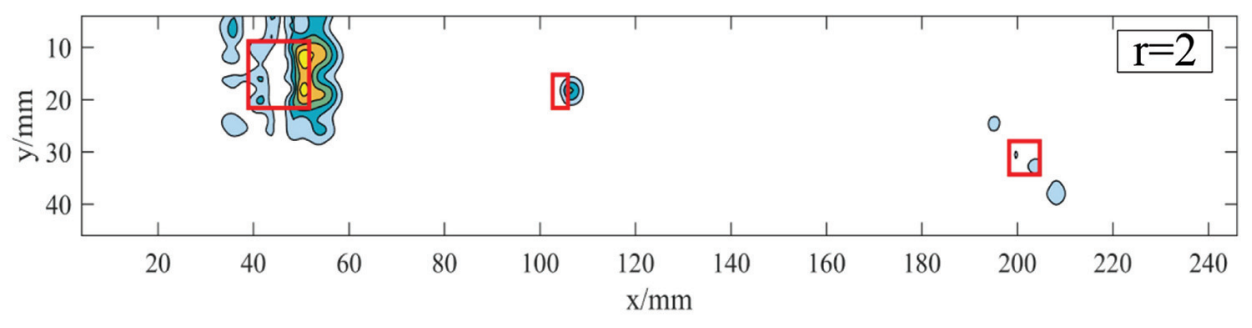

(b)
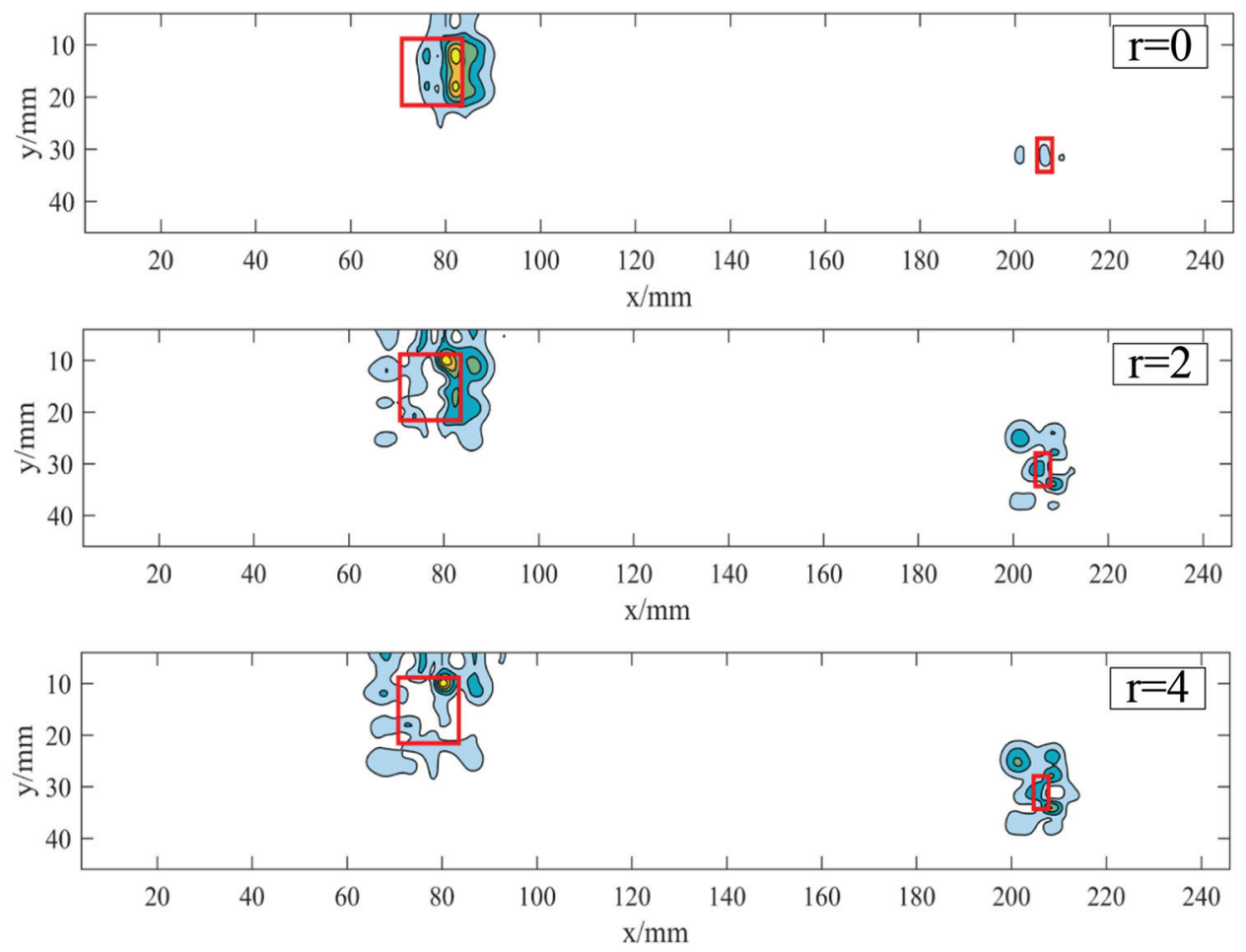

(c)

Figure 4. Results of $D I T_{r}$ : (a) MI, (b) M3, and (c) M2.

In order to suppress the global fluctuation and singularities caused by non-damage factors (boundary conditions, contact nodes, etc.), TEO is used to process damage index $D I_{r}$, and then the index $D I T_{r}$ can be obtained. The damage index $D I T_{r}$ for spatial sampling points can be defined as equation (8). In the work, damage index $D I T_{0}, D I T_{2}$ and $D I T_{4}$ are selected to identify damages of SPTCs

$$
D I T_{r}(k)=\left|D I_{r}^{2}(k)-D I_{r}(k-1) D I_{r}(k+1)\right|
$$

\section{Numerical simulation}

\section{Numerical model}

The sketch of the filled pyramid SPTC model being simulated is shown in Figure 1. Figure 1(a) gives the 


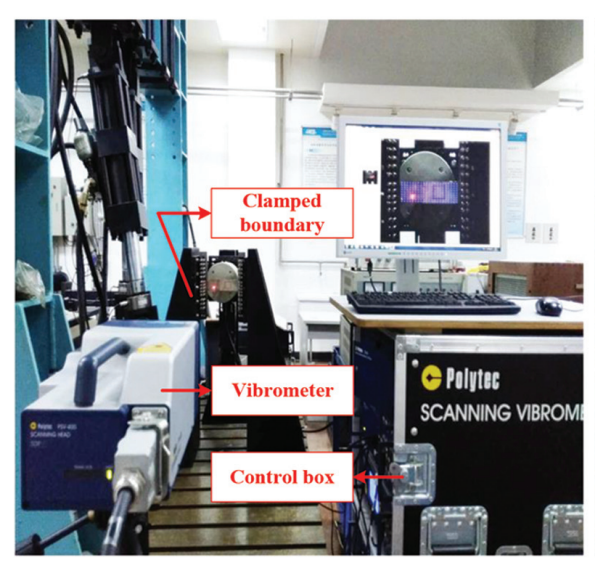

(a)

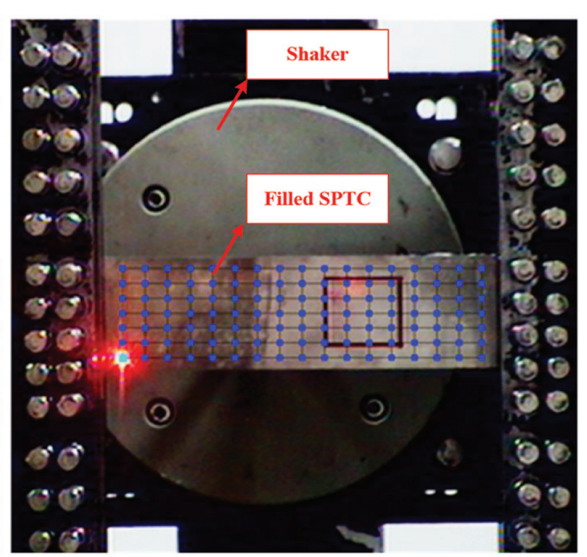

(b)

Figure 5. Experiment information: (a) experimental setup and (b) scanning points.

unit cell of pyramidal truss, where $h=4 \mathrm{~mm}$, $\theta=45^{\circ}, t=0.5 \mathrm{~mm}$. Figure 1(b) gives the illustration of low-density material filled SPTC. The filled material is silicone resin mixed with carbon powder, which is a typical lightweight ablation resistance material. Young's modulus and density of silicone resin mixed with carbon powder could be changed according to the proportion of carbon powder.

A typical damaged specimen is shown in Figure 2, and there are 7 units and 39 units along the $\mathrm{x}$ and $\mathrm{y}$ directions, respectively. The thickness of the face-sheet is $0.5 \mathrm{~mm}$. The SPTC is made of stainless steel with Young's modulus of $200 \mathrm{GPa}$, Poisson ratio of 0.3 , and mass density of $7800 \mathrm{~kg} / \mathrm{m}^{3}$. Young's modulus of the filled material is $100 \mathrm{MPa}$, Poisson ratio is 0.3 , and mass density is $1100 \mathrm{~kg} / \mathrm{m}^{3}$. The boundary condition is two sides clamped and two sides free (CCFF).

In the article, cell missing damages are considered and cases of single damage and multiple damages of different extents are simulated. The example of damaged model is given in Figure 2. Cases of single damage include half-cell missing (S1), one-cell missing (S2), and $2 \times 2$ cells missing (S3). And cases of multiple damages include one and half cells (M1); half-cell and $2 \times 2$ cells missing (M2); half-cell, one-cell, and $2 \times 2$ cells missing (M3). Damage locations in single-damage and multiple-damages cases are selected randomly. The first 10 order modes are used to calculate damage index $D I T_{r}$.

\section{Single-damage identification}

Identification of single damage with different extents is conducted. $D I T_{2}$ results are provided in Figure 3, and the red rectangle is the actual damage region. From Figure 3, it is understood that the proposed method could identify the single damage with different extents

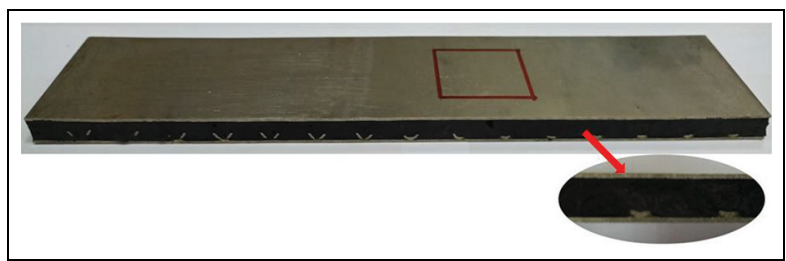

Figure 6. The damaged specimen: DS $2 \times 3$.

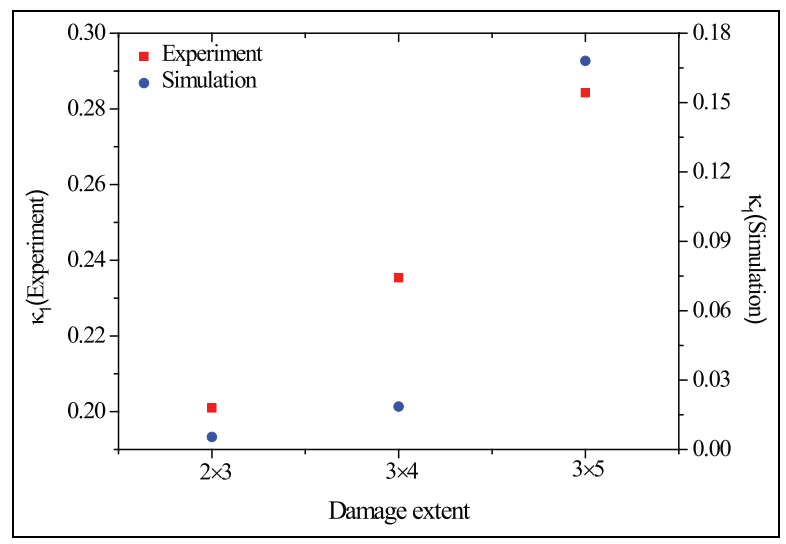

Figure 7. $\kappa_{1}$ results of simulations and experiments.

effectively, no matter where the damage is. Besides, as the extent of damages increases from S1 to S3, influences of damages on their surroundings also increases, demonstrating that the proposed damage index is sensitive to damage extent.

\section{Multiple-damages identification}

In order to verify the effectiveness of the method in multiple-damages identification, three damaged models 


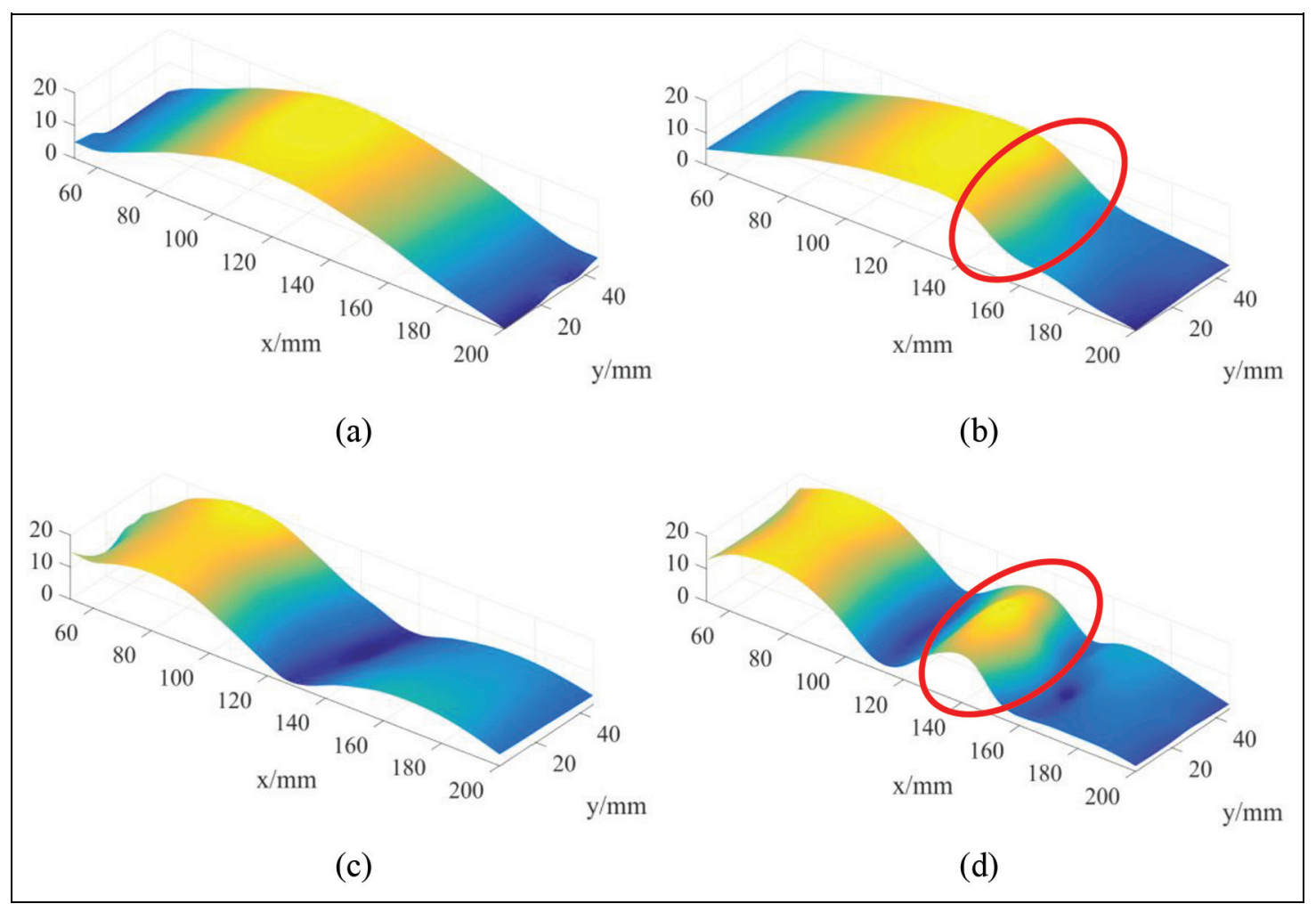

Figure 8. Tested mode shapes. (a) and (b): first mode shape of US and DS $2 \times 3$. (c) and (d): second mode shape of US and DS $2 \times 3$.

of filled pyramid SPTCs (named M1, M2, and M3) are used, and results are shown in Figure 4. The red rectangles represent the actual damage regions.

From Figure 4(a)-(c), all damages could be identified accurately, no matter whether there are two or three damages. To investigate the effect of weight coefficient on damage identification, results of M2 in the case of different $r$ are provided in Figure 4(c). From Figure 4(c), it is found that with the increasing of the weight coefficient, half-cell missing becomes more obvious. As small-extent damages normally have greater influences on higher-order modes than the lower-order modes. It reveals that high-order modes should be considered to locate damages of small extent. Besides, comparing results in the case of $r=0$ and $r=4$, it is also found that the extent identification is more accurate when $r=0$, whereas it is easier to locate the damage of small extent when $r=4$. Therefore, results in the case of different weight coefficient should be combined together to improve the accuracy of both damage location and damage extent identification.

Moreover, from Figure 4(a), although the damage extent of half-cell missing is smaller than that of onecell missing, the affected area of half-cell missing is much larger than that of one-cell missing. According to half-cell missing results in Figure 4(a)-(c), it is seen that the same extent damages at different locations would have different influences on structural modes or damage indexes. Besides, when the damage extent is the same, variations of modal information caused by damages at the boundary are normally larger than that caused by damages away from boundary. The result is in accordance with $\mathrm{Lu}$ et al., ${ }^{10}$ and Lou et al. ${ }^{35}$

\section{Experimental study}

\section{Experimental setup}

To verify the effectiveness of the proposed method and numerical model, experiments are also conducted. The experimental setup is shown in Figure 5(a). The specimens of metallic SPTCs with the filler are excited by a shaker (JZK-50). Two edges of the specimens are clamped. The excitation signal is generated by the control system of the laser Doppler vibrometer (Polytec, PSV-400) and amplified by a power amplifier (YE5872A) before input to the shaker. The frequency bandwidth of the excitation signal is set in the range of $1-4 \mathrm{kHz}$ with the resolution of $625 \mathrm{mHz}$.

The laser Doppler vibrometer is applied to measure the structural vibration response, and the scanning points are focused on the front face-sheet, as shown in 


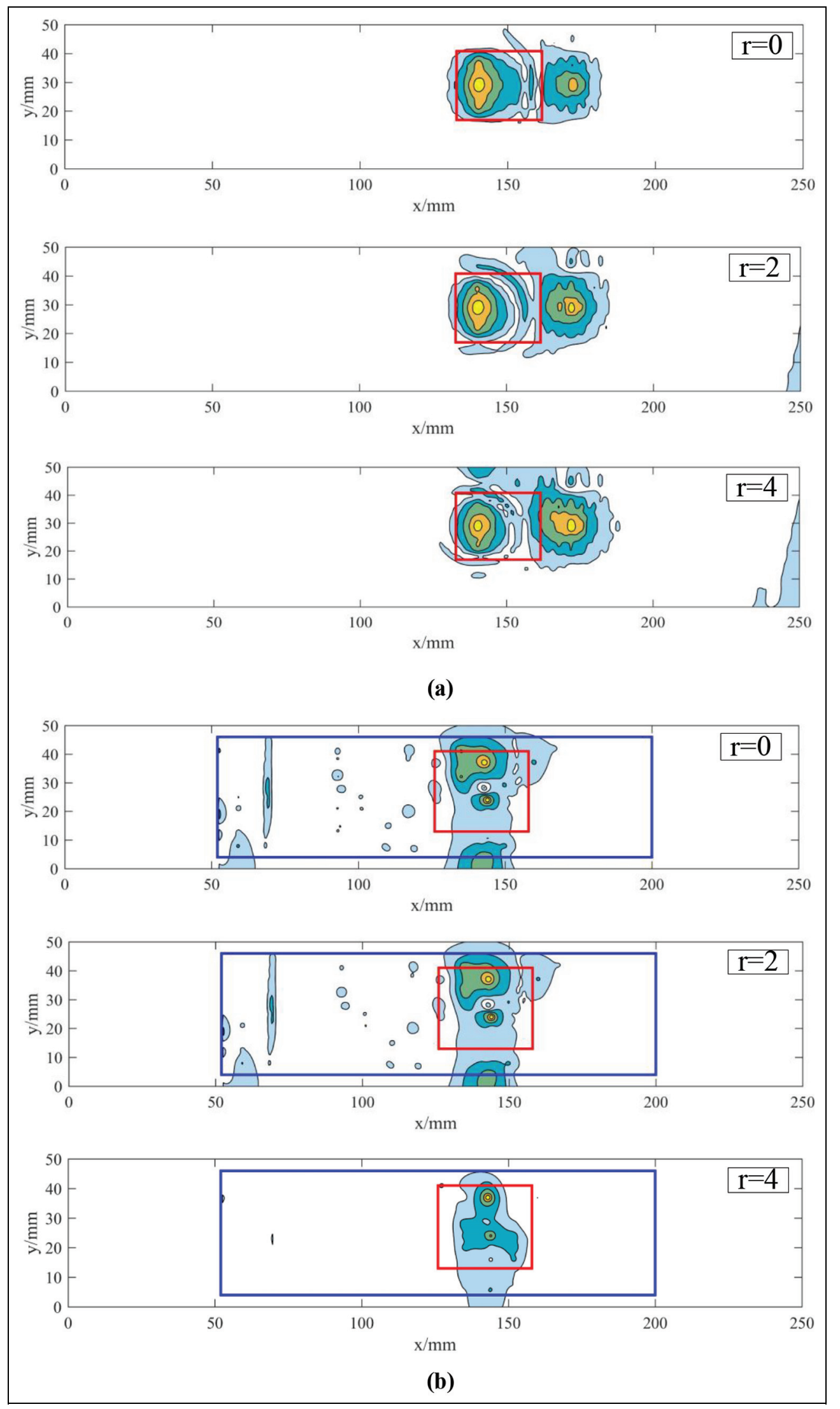

Figure 9. Identification results of DS $2 \times 3$ : (a) simulation and (b) experiment. 


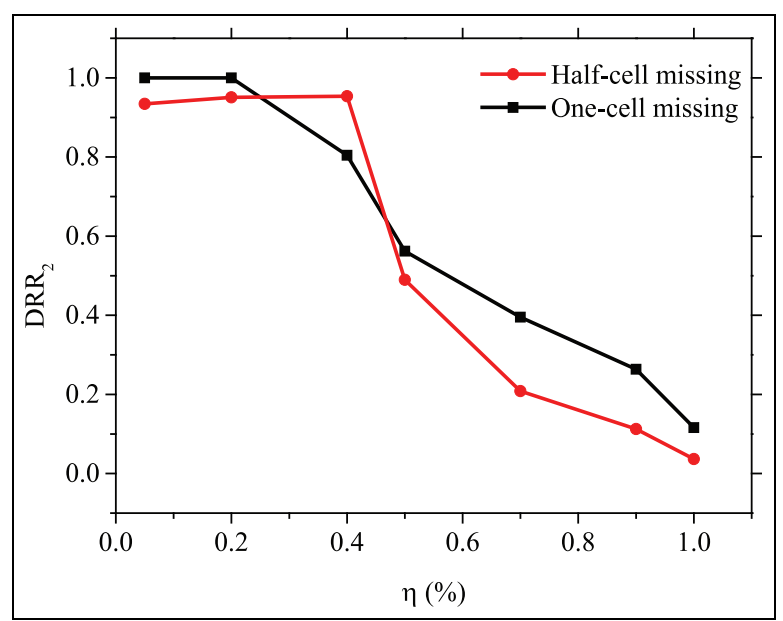

Figure I0. $D R R_{2}$ results.

Figure 5(b). The other side of the face-sheet is connected to the shaker by metallic bar. The obtained excitation and structural response signal are used to calculate the FRF to get structural modal information.

\section{The preparation of specimens}

In this work, the metallic pyramid truss cores with a relative density $\rho$ of about $3 \%$ are fabricated from 0.7 $\mathrm{mm}$ thick perforated stainless steel sheet by folding technique. The brazing technique is utilized to join the truss core with face-sheets. The dimension of the filled specimen is $50 \mathrm{~mm} \times 250 \mathrm{~mm}$. The thickness of the face-sheet is $0.9 \mathrm{~mm}$ and the truss core is $7 \mathrm{~mm}$. The lightweight ablation-resistant material, that is, silicone resin mixed with carbon powder, is filled into the prefabricated healthy and damaged pyramidal SPTCs, and the filler's density is $0.2 \mathrm{~kg} / \mathrm{m}^{3}$.

In the tests, cell missing damages are considered and truss core cells of the damaged location are cut out before brazing. Damages with different sizes and locations are considered. Undamaged specimen is named US, and the specimens with cells missing are named DS. The damage sizes include $2 \times 3$ cells missing, $3 \times 4$ cells missing, and $3 \times 5$ cells missing, named DS $2 \times 3$, DS $3 \times 4$, and DS $3 \times 5$, respectively. The specimen DS $2 \times 3$ is shown in Figure 6, and the red rectangle represents the damaged region.

\section{Experimental results}

The differential ratio of natural frequency $\kappa_{i}$ is defined to validate the numerical model

$$
\kappa_{i}=\frac{\left|f_{i}^{U}-f_{i}^{D}\right|}{f_{i}^{U}}
$$

where $f_{i}^{U}$ is the $i$ th natural frequency of the undamaged model, $f_{i}^{D}$ is the $i$ th natural frequency of the damage model.

The numerical models, having the same damage extents with the experimental models, are built, and the $\kappa_{1}$ results of both numerical simulations and experiments are presented in Figure 7. From Figure 7, although the values of $\kappa_{1}$ of experiments are different from those of simulations, the tendency of parameter $\kappa_{1}$ of numerical model is in accordance with that of experiment as the damage extent increases. Many factors contribute to the difference between simulation and experiment. For example, the boundary condition of numerical model is ideal and strict. However, the boundary condition of experimental model is not ideal, and it may loosen a little as the structure vibrates. The manufacturing process of the specimens may also induce errors.

The first two tested modes of US and DS $2 \times 3$ are provided in Figure 8. After the specimen is damaged, the vibration property of the local damaged zone is different from that of a US, as the red circles shown in Figure 8. After the mode shapes are obtained, the damage index is calculated based on the first 2 mode shapes, and results are shown in Figure 9.

The identification results of the most representative specimen, DS $2 \times 3$, are shown in Figure 9. The length of the restrict edge is $40 \mathrm{~mm}$; therefore, the real measured region is small and as the blue rectangle shown in Figure 9(b). To compare with numerical results, the $x$ and $y$ coordinate of the experimental results are extended. It is observed from Figure 9 that the damage could be identified effectively according to the numerical and experimental results. The difference between the simulations and experiments are induced by the boundary condition and the manufacturing process of the specimens.

\section{Discussion}

According to the dynamic theory, Young's modulus and density of the filler would affect dynamic property of the whole SPTC and then affect the damage identification. In the article, the influence of Young's modulus of the filler on damage identification is discussed. Filled SPTCs with half-cell missing and one-cell missing are employed to investigate the effect of the filler. In the work, when Young's modulus of the filler is 100, 400, $700,1000,1400,1700$, and $2000 \mathrm{MPa}$, the corresponding numerical model is analyzed, and the results are shown in Figures 10 and 11. In order to investigate the effect of Young's modulus of the filler quantitatively, the damage-recognition rate $D R R_{r}$ is defined as 


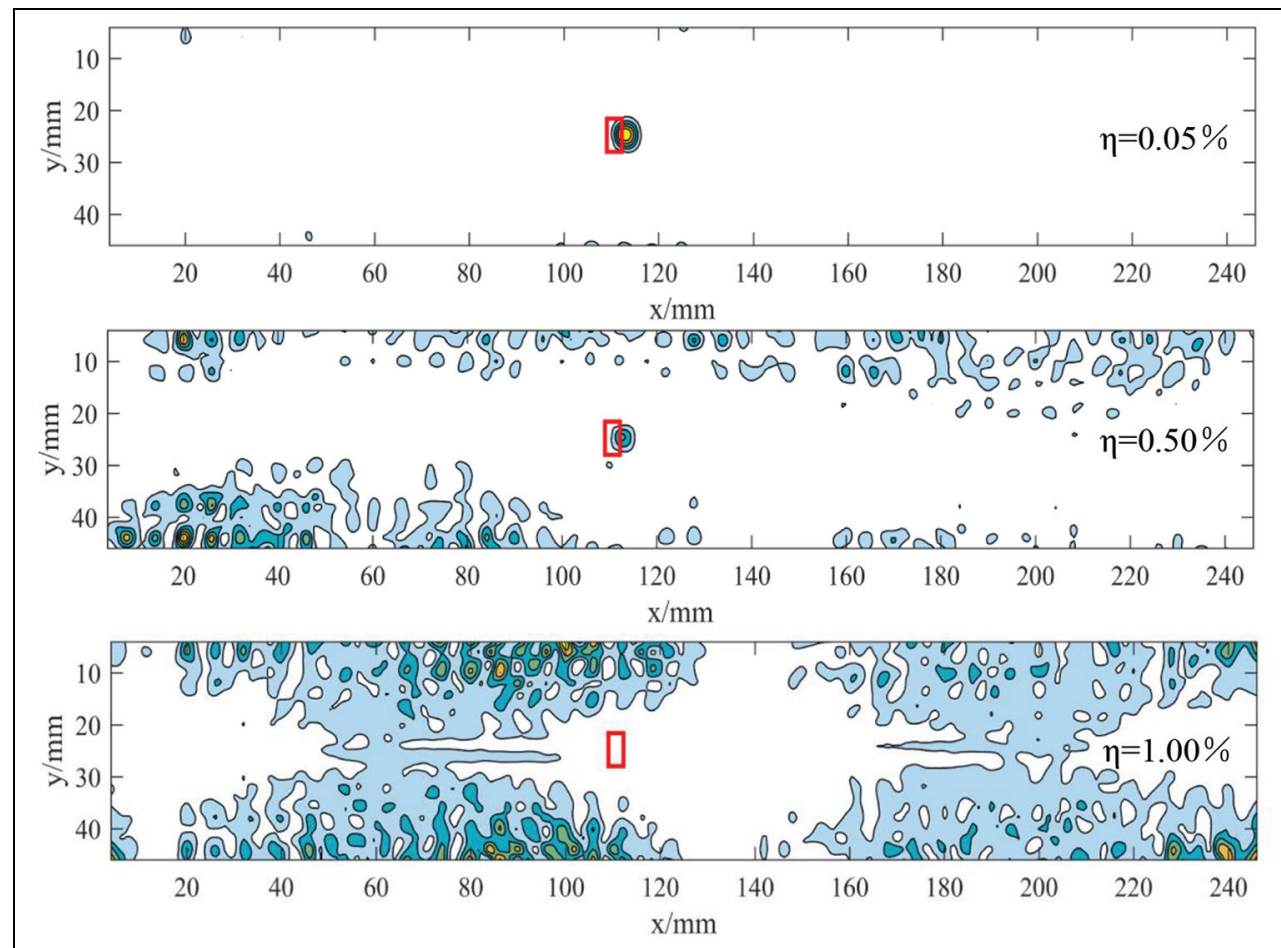

(a)
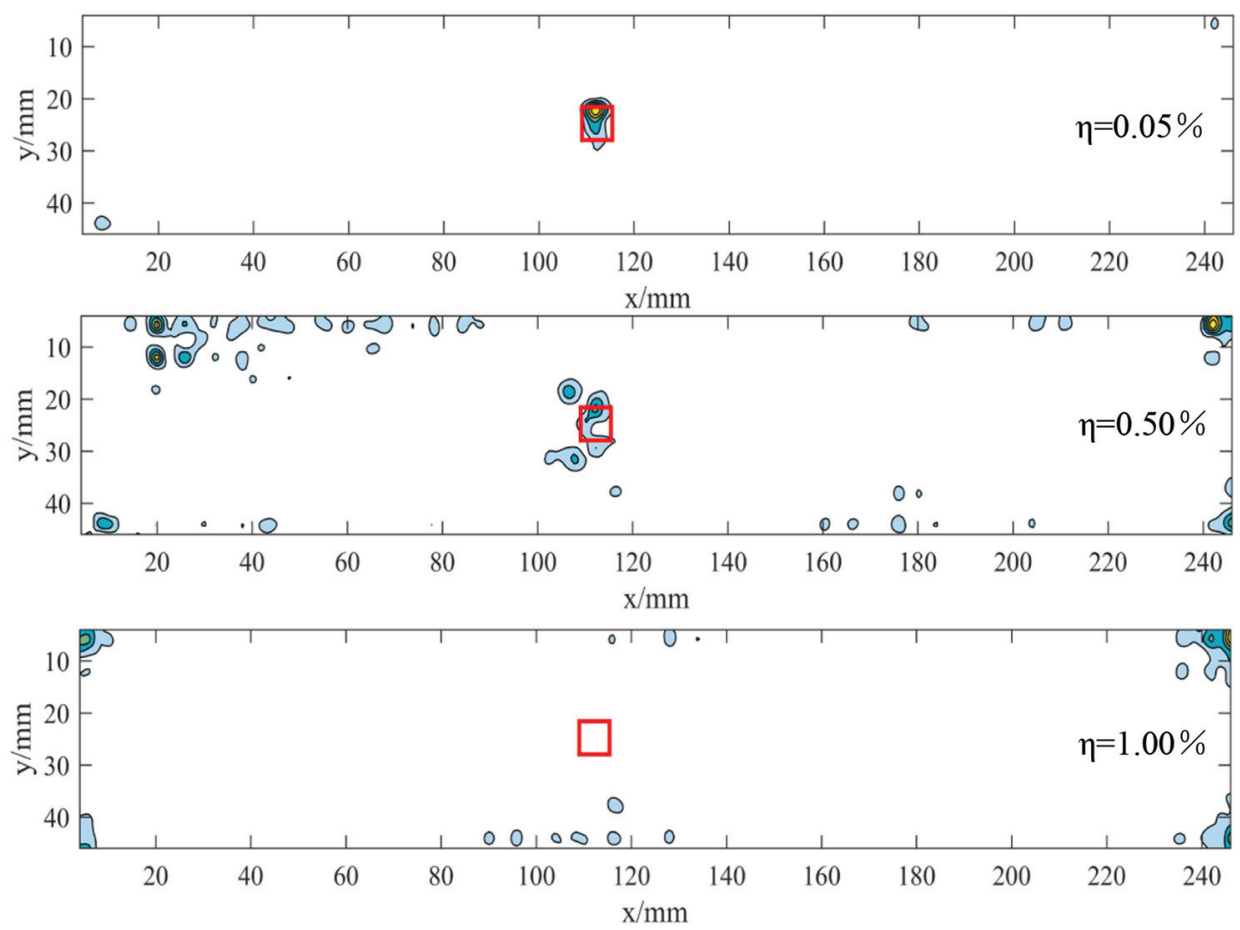

(b)

Figure I I. DIT 2 results of damaged specimens with different Young's modulus of the filler: (a) half-cell missing and (b) one-cell missing.

$$
D R R_{r}=\frac{D I T_{\max , r}^{D}}{D I T_{\max , r}^{W}}
$$

where $D I T W$ max, $r$ is the maximum $D I T_{r}$ value in the whole face-sheet. DITD max, $r$ is the maximum $D I T_{r}$ value in the damaged region. 
Parameter $\eta$ is defined as

$$
\eta=\frac{E_{f}}{E_{B M}}
$$

where $E_{f}$ and $E_{B M}$ are Young's modulus of filler and base material of SPTC, respectively.

$D R R_{2}$ results of the two damaged models, specimens with half-cell missing and one-cell missing are provided in Figure 10. It is clearly noted that $D R R_{2}$ decreases as Young's modulus of the filler increases. When the $D R R_{2}$ is around 1 , it demonstrates that the damage can be identified completely, as shown in the first line figures of Figure 11(a) and (b). When the $D R R_{2}$ is around 0.5 , the damage becomes invisible, as the figures in the case of $\eta=0.5 \%$ shown. When the $D R R_{2}$ is less than 0.5 , the real damage is completely unidentified. For the cases with half-cell and one-cell missing, when $\eta$ is larger than $0.5 \%$, the real damage could not be identified according to the proposed method.

When damage extent increases or the damage location changes, the critical $\eta$ may change. And more work will be done to investigate effect of material property of the filler on the damage identification of filled SPTC.

\section{Conclusion}

Low-density material-filled SPTC is the primary form of this type of sandwich panel during engineering practice. The filled functional material further enhances the performance of SPTC. However, it also presents new challenges in damage identification of filled SPTC. In this article, a vibration-based method is presented to detect damages in the filled SPTC. Weight coefficient $r$ is used to consider effects of damages on both highorder modes and low-order modes. Numerical simulations and experimental validations are conducted to assess effectiveness of the proposed method. Moreover, the effect of material property of the filler on the method is also discussed. Some conclusions can be drawn as follows:

1. The proposed method can identify both single damage and multiple damages with different extents effectively. The proposed weight coefficient $r$ can improve the effectiveness of the method, especially for identification of multiple damages or damages with small extent. For small-extent damages, results in the case of different weight coefficients must be combined together to improve the accuracies of both damage location and extent identification.

2. Local damage identification becomes more difficult as Young's modulus of the filler increases. For half-cell and one-cell damage, the critical $\eta$ is around $0.5 \%$. When the damage extent or damage location changes, the critical $\eta$ may also change. More works must be done to study the effect of Young's modulus of the filler.

\section{Declaration of conflicting interests}

The author(s) declared no potential conflicts of interest with respect to the research, authorship, and/or publication of this article.

\section{Funding}

The author(s) disclosed receipt of the following financial support for the research, authorship, and/or publication of this article: This research is supported by the National Natural Science Foundation of China (Grant nos.: 11472276, 11332011, and 11502268) and the National Defense Basic Scientific Research Program of China (Grant no.: JCKY2016130B009).

\section{ORCID iD}

Lingling Lu (iD) https://orcid.org/0000-0002-1296-0186

\section{References}

1. $\mathrm{Hg}$ A. Analysis design of structural sandwich panels. Oxford: Pergamon Press, 1969.

2. Chiras S, Mumm DR, Evans AG, et al. The structural performance of near-optimized truss core panels. Int $J$ Solids Struct 2002; 39(15): 4093-4115.

3. Wadley HNG, Fleck N and Evans AG. Fabrication and structural performance of periodic cellular metal sandwich structures. Compos Sci Technol 2003; 63(16): 2331-2343.

4. Zumpano G and Meo M. Damage detection in an aircraft foam sandwich panel using nonlinear elastic wave spectroscopy. Comput Struct 2008; 86(3): 483-490.

5. Panopoulou A, Loutas T, Roulias D, et al. Dynamic fiber Bragg gratings based health monitoring system of composite aerospace structures. Acta Astronaut 2011; 69(7): 445-457.

6. Evans AG, Hutchinson JW, Fleck NA, et al. The topological design of multifunctional cellular metals. Prog Mater Sci 2001; 46(3): 309-327.

7. Wang $\mathbf{J}$, Lu TJ, Woodhouse $\mathbf{J}$, et al. Sound transmission through lightweight double-leaf partitions: theoretical modelling. J Sound Vib 2005; 286(4): 817-847.

8. Yuan W, Song HW, Wang X, et al. Experimental investigation on thermal buckling behavior of truss-core sandwich panels. AIAA J 2014; 53(4): 948-957.

9. Yuan W, Wang X, Song HW, et al. A theoretical analysis on the thermal buckling behavior of fully clamped sandwich panels with truss cores. J Therm Stresses 2014; 37: 1433-1448.

10. Lu LL, Song HW and Huang CG. Effects of random damages on dynamic behavior of metallic sandwich panel with truss core. Compos Part B 2017; 116: 278-290. 
11. Yin S, Wu LZ, Ma L, et al. Hybrid truss concepts for carbon fiber composite pyramidal lattice structures. Compos Part B 2012; 43(4): 1749-1755.

12. Yazici M, Wright $\mathbf{J}$, Bertin D, et al. Experimental and numerical study of foam filled corrugated core steel sandwich structures subjected to blast loading. Compos Struct 2014; 110: 98-109.

13. Bin $\mathrm{H}, \mathrm{Bo} \mathrm{Y}, \mathrm{Yu} \mathrm{X}$, et al. Foam filling radically enhances transverse shear response of corrugated sandwich plates. Mater Design 2015; 77: 132-141.

14. Yazici M, Wright J, Bertin D, et al. Preferentially filled foam core corrugated steel sandwich structures for improved blast performance. J Appl Mech 2015; 82(6): 061005.

15. Ben Ammar I, Karra C, El Mahi A, et al. Mechanical behavior and acoustic emission technique for detecting damage in sandwich structures. Appl Acoust 2014; 86: 106-117.

16. Sikdar S, Ostachowicz W and Pal J. Damage-induced acoustic emission source identification in an advanced sandwich composite structure. Compos Struct 2018; 202: 860-866.

17. Wong YR, Du H and Pang X. Real-time electrical impedance resonance shift of piezoelectric sensor for detection of damage in honeycomb core sandwich structures. NDT\&E Int 2015; 76: 61-65.

18. Meola C, Boccardi S and Carlomagno GM. Nondestructive testing with infrared thermography. In: Meola $\mathrm{C}$, Boccardi S and Carlomagno GM (eds) Infrared thermography in the evaluation of aerospace composite materials. New York: Springer, 2017, pp. 85-125.

19. Colombo C, Harhash M, Palkowski H, et al. Thermographic stepwise assessment of impact damage in sandwich panels. Compos Struct 2018; 184: 279-287.

20. Ibarra-Castanedo C, Piau JM, Guilbert S, et al. Comparative study of active thermography techniques for the nondestructive evaluation of honeycomb structures. Res Nondestruct Eval 2009; 20(1): 1-31.

21. Li GY and Chattopadhyay A. Multi-dimensional signal processing and mode tracking approach for guided wave based damage localization in X-COR sandwich composite. Mech Syst Signal Pr 2018; 109: 134-149.

22. Chakraborty N, Rathod VT, Roy Mahapatra D, et al. Guided wave based detection of damage in honeycomb core sandwich structures. NDT\&E Int 2012; 49: 27-33.
23. Sikdar S and Banerjee S. Identification of disbond and high density core region in a honeycomb composite sandwich structure using ultrasonic guided waves. Compos Struct 2016; 152: 568-578.

24. Fan W and Qiao P. Vibration-based damage identification methods: a review and comparative study. Struct Health Monit 2011; 10(1): 83-111.

25. Katunin A. Vibration-based spatial damage identification in honeycomb-core sandwich composite structures using wavelet analysis. Compos Struct 2014; 118: 385-391.

26. Lestari W and Qiao P. Damage detection of fiberreinforced polymer honeycomb sandwich beams. Compos Struct 2005; 67(3): 365-373.

27. Tian SX, Chen ZM, Chen LL, et al. Numerical analyses on influence of damage configuration on vibration parameters for lattice sandwich plate. Int J Appl Electrom 2010; 33(3): 1565-1572.

28. Zhu KG, Chen MJ, Lu QH, et al. Debonding detection of honeycomb sandwich structures using frequency response functions. $J$ Sound Vib 2014; 333(21): 5299-5311.

29. Seguel F and Meruane V. Damage assessment in a sandwich panel based on full-field vibration measurements. $J$ Sound Vib 2018; 417: 1-18.

30. Li B, Li Z, Zhou J, et al. Damage localization in composite lattice truss core sandwich structures based on vibration characteristics. Compos Struct 2015; 126: 34-51.

31. Lu LL, Song HW, Yuan W, et al. Baseline-free damage identification of metallic sandwich panels with truss core based on vibration characteristics. Struct Health Monit 2016; 16(1): 24-38.

32. Lu LL, Song HW and Huang CG. Experimental investigation of unbound nodes identification for metallic sandwich panels with truss core. Compos Struct 2017; 163 : 248-256.

33. Yuan W, Wang JT, Song HW, et al. High-power laser resistance of filled sandwich panel with truss core: an experimental study. Compos Struct 2018; 193: 53-62.

34. Qi G and Ma L. Experimental investigation of composite pyramidal truss core sandwich panels with lightweight inserts. Compos Struct 2018; 187: 336-343.

35. Lou J, Wu L, Ma L, et al. Effects of local damage on vibration characteristics of composite pyramidal truss core sandwich structure. Compos Part B 2014; 62: 73-87. 